\title{
How many Hipparcos Variability-Induced Movers are genuine binaries? ${ }^{\star}, \star \star$
}

\author{
D. Pourbaix ${ }^{1,2, \star \star \star}$, I. Platais ${ }^{1,3,4}$, S. Detournay ${ }^{1,5}$, A. Jorissen ${ }^{1, \star \star \star}$, G. Knapp ${ }^{2}$, and V. V. Makarov ${ }^{3,6}$ \\ ${ }^{1}$ Institut d'Astronomie et d'Astrophysique, Université Libre de Bruxelles, CP 226, Boulevard du Triomphe, \\ 1050 Bruxelles, Belgium \\ 2 Department of Astrophysical Sciences, Princeton University, Princeton NJ 08543-1001, USA \\ 3 Universities Space Research Association, Division of Astronomy and Space Physics, 300 D Street SW, \\ Washington D.C. 20024, USA \\ ${ }^{4}$ Department of Physics and Astronomy, The Johns Hopkins University, 3400 North Charles Street, \\ Baltimore, MD 21218, USA \\ 5 Département de Physique, Université de Mons Hainaut, Avenue du Champ de Mars 8, 7000 Mons, Belgium \\ ${ }^{6}$ US Naval Observatory, Washington D.C., USA
}

Received 20 September 2002 / Accepted 5 December 2002

\begin{abstract}
Hipparcos observations of some variable stars, and especially of long-period (e.g. Mira) variables, reveal a motion of the photocenter correlated with the brightness variation (variability-induced mover - VIM), suggesting the presence of a binary companion. A re-analysis of the Hipparcos photometric and astrometric data does not confirm the VIM solution for 62 among the 288 VIM objects (21\%) in the Hipparcos catalogue. Most of these 288 VIMs are long-period (e.g. Mira) variables (LPV). The effect of a revised chromaticity correction, which accounts for the color variations along the light cycle, was then investigated. It is based on "instantaneous" $V-I$ color indices derived from Hipparcos and Tycho-2 epoch photometry. Among the 188 LPVs flagged as VIM in the Hipparcos catalogue, 89 (47\%) are not confirmed as VIM after this improved chromaticity correction is applied. This dramatic decrease in the number of VIM solutions is not surprising, since the chromaticity correction applied by the Hipparcos reduction consortia was based on a fixed $V-I$ color. Astrophysical considerations lead us to adopt a more stringent criterion for accepting a VIM solution (first-kind risk of $0.27 \%$ instead of $10 \%$ as in the Hipparcos catalogue). With this more severe criterion, only 27 LPV stars remain VIM, thus rejecting 161 of the $188(86 \%)$ of the LPVs defined as VIMs in the Hipparcos catalogue.
\end{abstract}

Key words. stars: binaries: close - astrometry - stars: variables: general

\section{Introduction}

The Hipparcos satellite (ESA 1997) obtained high-precision photometric and astrometric data for some 120000 stars, discovering in the process many new binary systems. In addition, a small number of possible binaries (288) were identified as variability-induced movers (hereafter VIM, Wielen 1996). The physical origin of this effect lies in a close binary companion of an intrinsic variable star, causing the photocenter of the system to move as the primary's luminosity varies. The Hipparcos

Send offprint requests to: D. Pourbaix,

e-mail: pourbaix@astro.ulb.ac.be

* Based on observations from the Hipparcos astrometric satellite operated by the European Space Agency (ESA 1997).

$\star \star$ Table 1 is also available in electronic form at the CDS, via anonymous ftp to

cdsarc.u-strasbg.fr $(130.79 .128 .5)$ or via

http://cdsweb.u-strasbg.fr/cgi-bin/qcat?J/A+A/399/1167

$\star \star \star$ Research Associate, F.N.R.S., Belgium. analysis allowed for the presence of such a companion, solving for its position with respect to the primary in addition to the position, parallax and proper motion of the system.

Although the VIM model is likely to be appropriate in some cases (Bertout et al. 1999), the results for some other systems are questionable. For instance, rather few VIM have been resolved by speckle interferometry even though they have been extensively observed by, for instance, the Washington team (Mason et al. 1999, 2001).

This situation led us to re-investigate the VIM solutions using an improved processing of the Hipparcos data. The entire available set of astrometric data was used, instead of only FAST data, as was the case with the preparation of the Hipparcos catalogue. This results in a decrease by $21 \%$ of the number of VIM solutions, especially the red ones (Sect. 2).

During processing for the Hipparcos catalogue, corrections were applied to the Hipparcos photometry and astrometry to correct for the intrinsic chromaticity of the optical system (i.e. the position of the diffraction spot on the detector depends upon 
the color of the star) and for the aging effects of the optics and detectors during the mission. These effects are especially important for red variables because of their extreme red colors and because their variability time scales are comparable to the duration of the mission. Moreover, red variables have changing colors, and this variation must be taken into consideration when applying the chromaticity correction. In the preparation of the Hipparcos catalogue, the reduction consortia nevertheless adopted a constant color in the chromaticity correction process. There is thus room for improvement using chromaticity corrections based on epoch colors, rather than on average colors. Platais et al. (2003) have devised a scheme to derive epoch colors based on Tycho-2 and Hipparcos epoch photometry. An improved chromaticity correction has then been applied to a sample of 188 long-period variable stars flagged as VIM in the Hipparcos catalogue (Sect. 4), using the epoch $V-I$ index derived from $\mathrm{Hp}-\mathrm{V}_{\mathrm{T} 2}$. Section 5 shows that a more stringent criterion (first-kind risk of only $0.27 \%$ ) for accepting VIM solutions seems appropriate based on astrophysical considerations.

\section{VIM model and first re-processing}

Besides the five astrometric parameters $\left(p_{1}, \ldots, p_{5}\right)$ used to model the apparent motion of the center of mass, the VIM model requires two additional parameters $\left(D_{\alpha^{*}}, D_{\delta}\right)$ to provide the direction along which the photocenter moves (Wielen 1996). It is assumed that the companion causing the VIM effect has a fixed relative position with respect to the variable star, i.e. there is no orbital motion on top of the back and forth motion of the photocenter along the segment joining the two components. These seven parameters are the unique minimizer of the least-square problem $\chi_{V}^{2}=\Xi^{\mathrm{t}} V^{-1} \Xi$ (ESA 1997, vol. 1, Sect. 2.3.5) where

$$
\begin{aligned}
\Xi= & \Delta v-\sum_{k=1}^{5} \frac{\partial v}{\partial p_{k}} \Delta p_{k} \\
& -\left(\frac{\partial v}{\partial p_{1}} D_{\alpha^{*}}+\frac{\partial v}{\partial p_{2}} D_{\delta}\right) 10^{0.4\left(H p_{\text {tot }}-H p_{\text {ref }}\right)}
\end{aligned}
$$

is a vector with as many components as there are observations, and $H p_{\text {tot }}$ is the total, instantaneous magnitude of the system, whereas $H p_{\text {ref }}$ is an arbitrary reference magnitude. $\Delta v$ is the abscissa residual along a reference great circle. The displacements $D_{\alpha^{*}}$ and $D_{\delta}$ are tied to that reference magnitude. Unlike most of the other Hipparcos products, the VIM solution was built on FAST data only (Lindegren et al. 1997).

Before the effect of the chromaticity correction is investigated in detail (Sect. 4), let us first try to reproduce the original Hipparcos results using the astrometric and photometric data as they were released with the catalogue.

As far as the Intermediate Astrometric Data (IAD) are concerned, identifying those which have been used to produce the original VIM solution is straightforward since each IAD is flagged with $\mathrm{F}$ or $\mathrm{N}$, according to whether FAST or NDAC used it. However, not all of them were actually used. The same rejection criterion as the one used by the consortia is adopted, namely an abscissa is rejected if its residual is larger than three times its formal error (as given in field IA9).

The situation is a bit more obscure for the photometric data given in the Epoch Photometry Annex. There might be several observations corresponding to the very same astrometric data. Furthermore, there is a 9-bit flag associated with each observation that tells whether the magnitude can be trusted, and which consortium used it. According to the Hipparcos explanatory notes (ESA 1997, vol. 1, p. 220), if any of bits 3-8 are set, the data are likely to be unreliable. However, it appears that we cannot strictly follow this criterion since there is one star (HIP 118188 = R Cas) for which all photometric data are marked as unreliable. Thus we relaxed this criterion and accepted a photometric observation even with bit number 4 set, i.e. possible interfering object in either field of view. The median of the adopted magnitudes is taken as $H p_{\text {tot }}$ for the corresponding IAD (Eq. (2)). If no magnitude is available for a given IAD, the reference magnitude $\left(H p_{\text {ref }}\right)$ is adopted instead.

The statistical significance of the VIM model is assessed through $F_{D}$ :

$$
F_{D}^{2}=\left(\begin{array}{ll}
D_{\alpha^{*}} & D_{\delta}
\end{array}\right)\left(\begin{array}{cc}
\sigma_{D_{\alpha^{*}}}^{2} & \rho_{D} \sigma_{D_{\alpha^{*}}} \sigma_{D_{\delta}} \\
\rho_{D} \sigma_{D_{\alpha^{*}}} \sigma_{D_{\delta}} & \sigma_{D_{\delta}}^{2}
\end{array}\right)^{-1}\left(\begin{array}{c}
D_{\alpha^{*}} \\
D_{\delta}
\end{array}\right) .
$$

The VIM model is accepted if $F_{D}>2.15$ which corresponds to a $10 \%$ chance of a false detection for a Gaussian error distribution. This criterion is the same as in the original processing.

Even though all these criteria are chosen to match as closely as possible those adopted during the original reduction, the VIM solution based on FAST data is confirmed for only 234 systems, as compared to 288 in the Hipparcos catalogue (Fig. 1a). We checked that the number of accepted VIM solutions depends only barely upon the criterion used for selecting the photometric data set. This selection process can thus not be the cause of the decrease in the number of accepted VIM solutions with our reprocessing. Neither is the IAD outlier rejection process, since we checked that the number of outlying IAD rejected by the present processing (171/8689 for FAST, and 189/9050 for NDAC) is not larger than in the original processing (about 200 for $\mathrm{FAST}^{1}$ ). The only explanation we can think of to account for the larger number of stars flagged as VIM in the Hipparcos catalogue is that the Hipparcos VIM solutions were derived using slightly different data from that published in the catalogue.

If the object is a genuine VIM, the NDAC data should also show the VIM effect. Without changing the threshold on $F_{D}$, one can further screen the VIM solutions by requiring that the solution based on the whole data set (FAST \& NDAC) fulfills it. By doing so, 8 more objects are discarded, resulting in a total decrease of $21 \%$ with respect to the original number of VIM (Fig. 1a).

\footnotetext{
1 This number is estimated from field $\mathrm{H} 29(F 1)$ giving the fraction of rejected data points, multiplied by the number of NDAC observations, and summing up over the whole sample. An inconsistency is sometimes observed in the catalogue in this respect, since $F 1$ may be different from zero, although none of the IAD is flagged as rejected!
} 


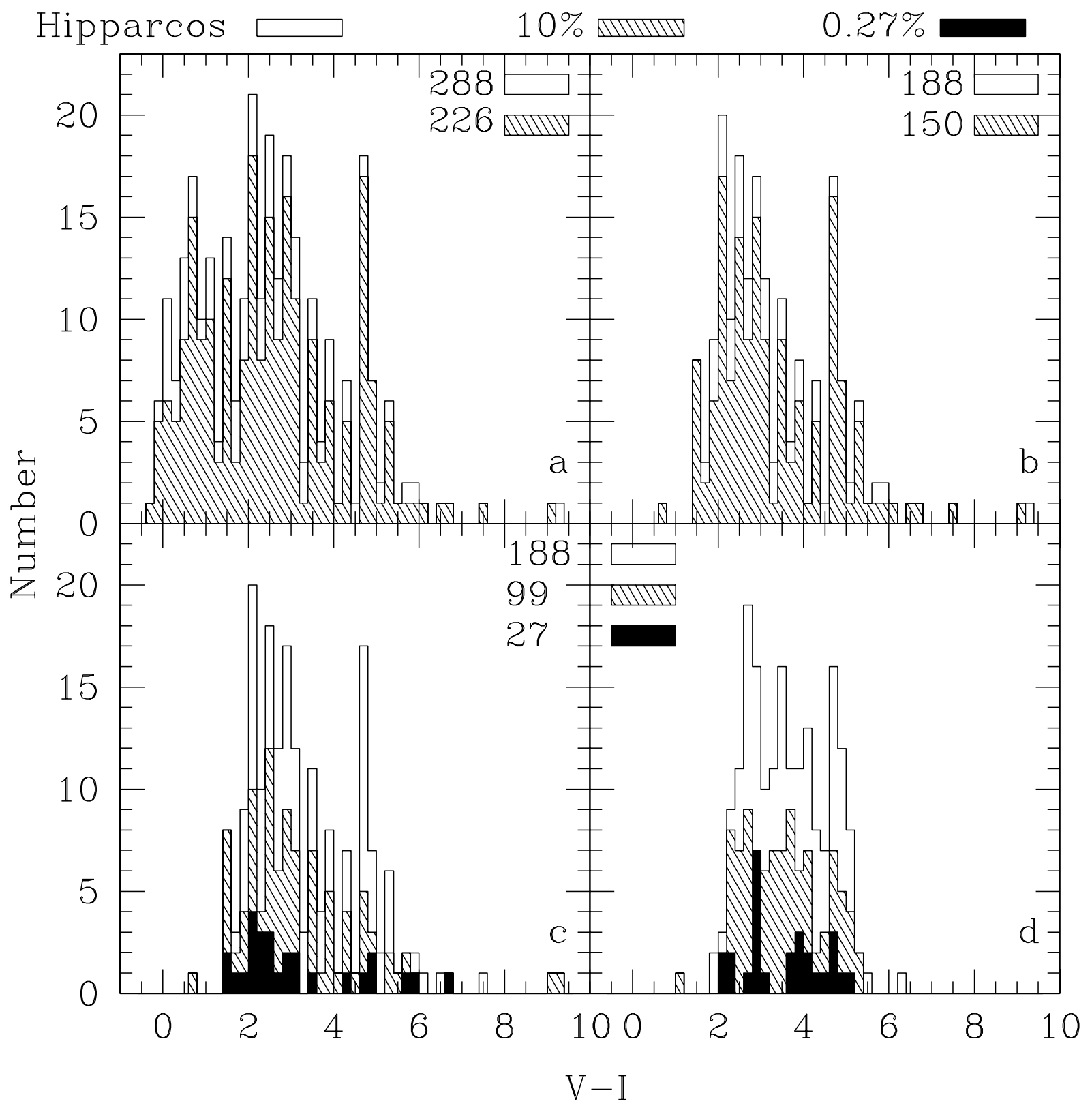

Fig. 1. Panel a): Distribution of the $(V-I)_{\mathrm{H} 75}$ indices for VIM solutions obtained with a processing similar to that of Hipparcos but applied to the combined FAST \& NDAC data set. The histogram labeled "Hipparcos" corresponds to the VIM solutions from the Hipparcos catalogue. Panel b): Same as a) for the 188 VIM for which epoch $V-I$ indices are available (i.e., for M, S, and C spectral type stars). The VIM solutions are adopted at the $10 \%$ confidence level $\left(F_{D}>2.15\right.$ in Eq. (2)). The $V-I$ used in those two panels (both for processing and plotting) is from field H75. Panels c) and d): Same as b), but after applying a processing allowing for time-varying $V-I$ indices and chromaticity corrections (Sect. 4). The shaded and black histograms correspond to VIM solutions accepted at the $10 \%$ confidence level $\left(F_{D}>2.15\right.$ in Eq. $\left.(2)\right)$ or $0.27 \%$ confidence level $\left(F_{D}>3.44\right)$, respectively. In panel c), the histogram is based on $\mathrm{H} 75$ whereas the median of the instantaneous $V-I$ index is used in panel d).

\section{Revised chromaticity correction}

Each astrometric measurement is affected by the color of the star, since it controls the position of the diffraction spot on the Hipparcos detector (see Sect. 11.3 of vol. 3, ESA 1997). Each star observed by Hipparcos has therefore been assigned a $V-I$ color (or more precisely, $V-I_{\mathrm{C}}$ in the Cousins system). This color index is in turn used to compute the chromaticity correction (Sects. 16.3 and 16.4 of vol. 3, ESA 1997).
The two reduction consortia worked independently and concurrently towards the preparation of the Hipparcos catalogue. They used different relations for the chromaticity correction. NDAC adopted (Eq. (16.8), vol. 3, ESA 1997):

$v^{\mathrm{o}}=v^{\mathrm{c}}+\left[Q_{N}+\Gamma_{23}+\Gamma_{24}(t-1991.25)\right] \frac{\lambda_{\mathrm{eff}}-550 \mathrm{~nm}}{550 \mathrm{~nm}}+\eta$

where $v^{\mathrm{o}}$ and $v^{\mathrm{c}}$ are the observed and computed great-circle abscissae, $Q_{N}$ is the a priori chromaticity constant for the orbit $N$ (kindly provided to us by L. Lindegren), $t$ is the epoch of the 


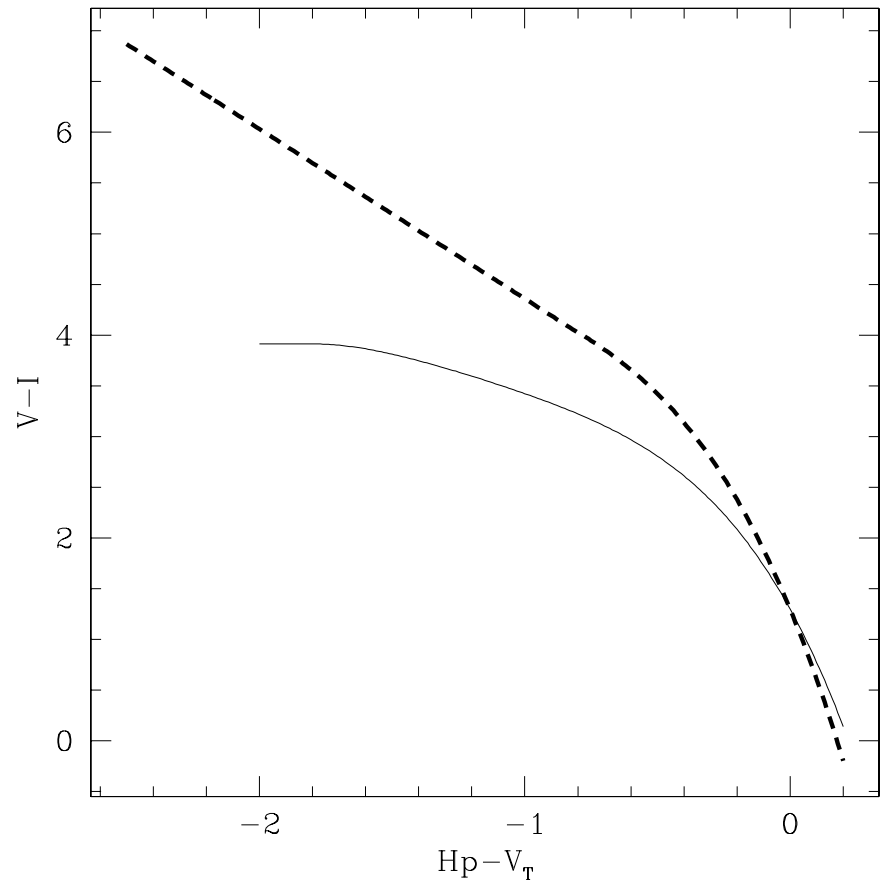

Fig. 2. The adopted transformation between $H p-V_{\mathrm{T}}$ and $V-I$, specific to carbon stars (solid line) or oxygen stars (dashed line), according to Platais et al. (2003).

orbit (expressed in years) and $\eta$ is the residual noise. The chromatic parameters are

$\Gamma_{23}=0.049 \pm 0.004$ mas,

$\Gamma_{34}=0.010 \pm 0.005 \mathrm{mas} / \mathrm{yr}$,

$\lambda_{\text {eff }}$ is the effective wavelength of a given stellar spectrum as observed through the $H p$ bandpass, and can be expressed as a function of $V-I$.

FAST accounted for the chromaticity effect through (Eqs. (16.12)-(16.18), vol. 3, 1997):

$$
\begin{aligned}
v^{\mathrm{o}}= & v^{\mathrm{c}}-0.3221[(V-I)-0.5] \\
& +0.1226[(V-I)-0.5]^{2} \\
& -0.0622[(V-I)-0.5](t-1991.25) .
\end{aligned}
$$

Note that, in the original processing, $V-I$ is kept constant in the reduction of any given star even though the star might be variable, with its color changing accordingly. The $V-I$ value used by the consortia for the astrometric processing is given in field $\mathrm{H} 75$ of the Hipparcos catalogue.

Unlike the situation prevailing during the preparation of the Hipparcos catalogue, now we have access to the Tycho-2 data (Høg et al. 2000), providing photometry for individual transits, as well as to recent ground-based $V-I$ observations for selected red stars. A detailed analysis of all these photometric data (Platais et al. 2003) yields the transformation relations from $H p-V_{\mathrm{T}}$ to $V-I$ given in Fig. 2, which allow us to find $V-I$ for each epoch $H p$.

\section{New processing}

As mentioned in Sect. 3, a constant $V-I$ index was assumed for each object by the Hipparcos reduction consortia, even when this object is a variable star and thus $V-I$ is likely to change too. It is therefore possible that many VIM solutions are just artifacts resulting from the neglected $V-I$ variations. In order to check this hypothesis, we reprocessed the VIM stars and take the $V-I$ variations into account when computing the chromaticity correction. The relation between $H p$ and $V-I$ derived by Platais et al. (2003) and calibrated on red stars has been used for that purpose, and, from now on, we limit our investigations to those 188 red VIM stars for which the $V-I$ indices can be computed from the data provided by Platais et al. (2003). To provide an adequate comparison, these 188 red VIM have first been reprocessed in the Hipparcos manner, as described in Sect. 2, with the results displayed in Fig. 1b. Only 150 VIMs are obtained in this Hipparcos-like reprocessing.

The results for the reprocessing with variable $V-I$ indices are presented in Table 1 and Figs. 1c, d. Only 99 stars out of 188 (or 53\%) have a VIM solution significant at the $10 \%$ level (corresponding to $F_{D}>2.15$ ). This dramatic reduction is a clear indication that many VIM solutions in the Hipparcos catalogue are spurious. They result either from the assumed constancy of $V-I$, as discussed above, or from the inappropriate mean $V-I$ index adopted by the Hipparcos reduction consortia (field H75). Table 2 collects 19 stars flagged as VIM in the Hipparcos catalogue, and with very red $V-I$ indices, namely $(V-I)_{\mathrm{H} 75}>5.0$. Most of these very large values are not confirmed by the $\left(V-I, H p-V_{\mathrm{T} 2}\right)$ color transformation derived by Platais et al. (2003). Interestingly, that the newly derived $V-I$ indices often agree well with the $(V-I)_{\mathrm{H} 40}$ indices derived at the end of the mission confirms that the $(V-I)_{\mathrm{H} 75}$ value adopted by the reduction consortia to derive the chromaticity correction was incorrect. Among these 19 stars, only 3 (HIP 2215, HIP 68815 and HIP 93605) remain VIM objects after our reprocessing. HIP 93605 (=HD 177017) is indeed a visual binary, with a $V=13.3$ companion located at 1.' 1 (Proust et al. 1981) (See also Sect. 5.3.2).

In Table 1, many of the stars flagged as VIM in the Hipparcos catalogue, and reprocessed with a single-star solution, keep almost exactly the same parallax as the Hipparcos one (some examples are HIP 5559 = RU Cas, HIP 13502 = R Hor, HIP $25412=\mathrm{R}$ Oct, ...), another clear indication that most of the Hipparcos VIM solutions were artifacts. In a few cases (HIP 1834, 1901, 65835, 77027, 99082), the Hipparcos parallax and the reprocessed parallax are largely different, thus calling for independent ways to check the new parallaxes. This is done in the next section, where those discrepant cases are specifically discussed.

Owing to the astrophysical importance of Mira variables, Table 1 also provides an estimator of the quality of the parallax, regardless of the model adopted. For this purpose, we evaluate not only the $\chi^{2}$ of the adopted model (denoted $\chi_{V}^{2}$, see Eq. (2)) but also the $\chi^{2}$ (denoted $\chi_{0}^{2}$ ) for the same model but with $\varpi=0$. The significance of the parallax can then be evaluated with an $F$-test:

$\hat{F}=\frac{N-p}{1} \frac{\chi_{0}^{2}-\chi_{V}^{2}}{\chi_{V}^{2}}$ 
Table 1. 188 red VIM from the Hipparcos catalogue, for which epoch $(V-I)$ indices are available from the $\left(V-I, H p-V_{\mathrm{T} 2}\right)$ color transformation (Platais et al. 2003), reprocessed with an improved chromaticity correction. The label "5" (standing for 5-parameter, single-star solution) in column "Sol." means that our reprocessing does not confirm the VIM nature of the object, at the $0.27 \%$ confidence level $\left(F_{D}<3.44\right.$ in Eq. $\left.(2)\right)$. The variability type is from Kholopov et al. (1998). $1-\alpha$ is the confidence level that the parallax is different from zero (Eq. (6)).

\begin{tabular}{|c|c|c|c|c|c|c|c|c|c|c|c|c|c|}
\hline HIP & CVS & Var & HIP (mas) & Sol. & $\varpi$ (mas) & & HIP & GCVS & Var & $\varpi_{\mathrm{HIP}}$ (mas) & Sol. & $\varpi$ (mas) & $\alpha$ \\
\hline 703 & & $\mathrm{M}$ & 50 & 5 & & 62 & & V Aur & 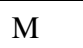 & & 5 & .61 & $\overline{0}$ \\
\hline 781 & Cas & & & V & & 13 & & & & & V & & 1 \\
\hline 1236 & Scl & $1 v$ & & 5 & & 25 & & & $\mathrm{~L}$ & & 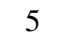 & & 4 \\
\hline 1593 & X And & & & 5 & & 9 & & RV Pup & & & . & & 33 \\
\hline 1834 & T Cas & $\mathrm{M}$ & & 5 & 90 & 2 & & GY Mon & & & 5 & & 11 \\
\hline 1901 & And & $\mathrm{M}$ & & 5 & & 10 & & R Gem & $\mathrm{M}$ & & 5 & $=1.56$ & 9 \\
\hline 2180 & Q And & & & 5 & & 42 & & & & & 5 & & 48 \\
\hline 2215 & & & & V & 9.07 & 0 & & Y Lyn & $\mathrm{L}$ & & V & & 0 \\
\hline 2546 & & $\mathrm{~N}$ & & 5 & & 95 & & & & & 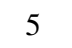 & & 28 \\
\hline 2880 & & & & 5 & & 46 & & & M & & V & & 99 \\
\hline 4284 & W Cas & $\mathrm{M}$ & & 5 & & 77 & & & M & & 5 & & 91 \\
\hline 4406 & $\mathrm{X}$ & & & 5 & & 2 & & $\mathrm{NC}$ & $\mathrm{L}$ & & 5 & & 6 \\
\hline 5559 & & & & 5 & & 1 & & & & & 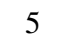 & & 55 \\
\hline & & & & 5 & & 6 & & & & & $J$ & & 53 \\
\hline 6952 & & & & 5 & & 0 & & & M & & 5 & & 5 \\
\hline 8025 & & & & 5 & & 50 & & & & & 5 & & 7 \\
\hline 8034 & V366 & $\mathrm{L}$ & & 5 & & 44 & & & & & V & & 0 \\
\hline 9767 & & & & 5 & & 2 & & & & & & & 0 \\
\hline 10904 & & & & 5 & & 44 & & & & & 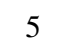 & & 63 \\
\hline 11093 & S Per & & & V & & 87 & & & & & 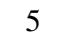 & & 27 \\
\hline 11 & & & & 5 & & 82 & & & & & 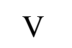 & & 0 \\
\hline 11 & & $\mathrm{~L}$ & & 5 & & 0 & & & & & 5 & & 42 \\
\hline & & $\mathrm{N}$ & & 5 & & 82 & & & & & 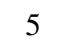 & & 68 \\
\hline 12 & & $\mathrm{U}$ & & 5 & & 0 & & & & & 5 & & 0 \\
\hline 13502 & & $\mathrm{M}$ & & 5 & & 2 & & & & & 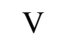 & & 0 \\
\hline 14042 & & $\mathrm{~N}$ & & 5 & & 63 & & & & & & & 0 \\
\hline 14229 & & & & 5 & & 0 & & & & & 5 & & 17 \\
\hline & & & & 5 & & 74 & & & $\mathrm{~L}$ & & 5 & & 4 \\
\hline & & $\mathrm{L}$ & & 5 & & 0 & & & M & & 5 & & 30 \\
\hline & & & & 5 & & 10 & & & & & 5 & & 0 \\
\hline 166 & & $\mathrm{~N}$ & & 5 & & 9 & & & & & 5 & & 3 \\
\hline & & & & 5 & & 22 & & $\mathrm{H}$ & & & 5 & & 15 \\
\hline & & & & 5 & & 0 & & & $\mathrm{~T}$ & & 5 & & 49 \\
\hline & & & & 5 & & 5 & & & & & 5 & & 10 \\
\hline 21479 & & & & 5 & & 0 & & & $x$ & & 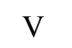 & & 2 \\
\hline 21766 & & $\mathrm{~N}$ & & 5 & & 85 & & & & & J & & 77 \\
\hline 22 & & $\mathrm{~N}$ & & 5 & & 41 & 57 & & & & 5 & & 80 \\
\hline & & M & & 5 & & 53 & & & & & 5 & & 35 \\
\hline 22552 & & & & 5 & & 97 & & & & & 5 & & 30 \\
\hline & & I & & 5 & & 0 & & & & & 5 & & $\infty$ \\
\hline 22670 & & & & 5 & & 65 & & & & & 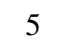 & & 3 \\
\hline 23200 & & $\mathrm{BY}+\mathrm{UV}$ & & 5 & & 0 & & $\mathrm{U}$ & & & 5 & & 66 \\
\hline 23520 & EL A & & & V & & 1 & 62401 & RU Vir & M & & 5 & & 17 \\
\hline & & 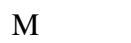 & & 5 & & 65 & & & 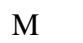 & & 5 & & 31 \\
\hline 23680 & W Ori & & & 5 & & 1 & & & . & & 5 & & 1 \\
\hline & & M & & 5 & & 0 & & Com & & & 5 & & 1 \\
\hline 27181 & & & & 5 & & 24 & 64768 & FH Vir & & & 5 & & 5 \\
\hline 27286 & $\mathrm{SC}$ & & & 5 & & 70 & 64778 & UY Ce & & & 5 & & 16 \\
\hline & & $\mathrm{L}$ & & 5 & & 72 & & & I & & 5 & & $x$ \\
\hline 28166 & BO Ori & $\mathrm{SF}$ & & 5 & & 3 & & & & & 5 & & 0 \\
\hline & S Vir & $\mathrm{M}$ & 4.29 & 5 & & 42 & 94162 & SZ Dra & $\mathrm{L}$ & 76 & 5 & $3.02 \pm 0.69$ & 0 \\
\hline & & M & & 5 & $2 \pm 0.84$ & 92 & 94224 & V3954 S & LB: & $3.91 \pm 1.24$ & 5 & $4.10 \pm 1.04$ & 1 \\
\hline 67410 & $\mathrm{R} \mathrm{Cl}$ & M & $-0.49 \pm 1.47$ & V & $-2.33 \pm 1.27$ & 9 & 94706 & T Sgr & M & $-31.67 \pm 9.28$ & V & $-16.14 \pm 8.52$ & 0 \\
\hline
\end{tabular}


Table 1. continued.

\begin{tabular}{|c|c|c|c|c|c|c|c|c|c|c|c|c|c|}
\hline HIP & GCVS & Var & $\varpi_{\mathrm{HIP}}$ (mas) & Sol. & $\varpi$ (mas) & $\alpha$ & HIP & GCVS & Var & $\varpi_{\mathrm{HIP}}(\mathrm{mas})$ & Sol. & $\varpi$ (mas) & $\alpha$ \\
\hline 67419 & W Hya & SRA & $8.73 \pm 1.09$ & 5 & $12.85 \pm 0.99$ & 0 & 94738 & R Sgr & M & $1.19 \pm 1.90$ & 5 & $2.59 \pm 1.45$ & 20 \\
\hline 67626 & RX Cen & M & $-17.92 \pm 7.60$ & 5 & -8.59 & 31 & 95173 & T Sge & L & 2.22 & 5 & .15 & 8 \\
\hline 68815 & $\theta$ Aps & SRB & $9.93 \pm 0.64$ & V & $10.36 \pm 0.63$ & 0 & 95676 & SW Tel & M & $-5.39 \pm 6.58$ & $\mathrm{~V}$ & $-5.50 \pm 5.74$ & 35 \\
\hline 68837 & U Cir & SR & $-1.92 \pm 1.51$ & 5 & $-2.31 \pm 1.35$ & 17 & 96836 & TT Cyg & SRB & $1.96 \pm 0.80$ & 5 & 1.36 & 14 \\
\hline 70885 & V Boo & SRA & $4.06 \pm 0.93$ & 5 & $4.86 \pm 0.83$ & 0 & 97586 & GY Aql & SR & 53 & 5 & .26 & 14 \\
\hline 70969 & Y Cen & I & 3.1 & 5 & 5.57 & 0 & 97629 & $\chi \mathrm{Cyg}$ & $\mathrm{N}$ & .36 & 5 & 1.00 & 0 \\
\hline 71802 & RW Boo & $\mathrm{L}$ & $3.09 \pm$ & 5 & $2.48 \pm 1.00$ & 5 & 97644 & $\mathrm{~T}$ Pav & $\mathrm{N}$ & $-0.54 \pm 1.43$ & 5 & .20 & 52 \\
\hline 75143 & $\mathrm{~S} \mathrm{CrB}$ & M & $1.90=$ & 5 & $2.40 \pm 1.17$ & 15 & 98031 & $\mathrm{~S}$ Pav & SRA & $2.91 \pm 1.77$ & 5 & 1.09 & 1 \\
\hline 75393 & RS Lib & M & $1.74 \pm 2.06$ & 5 & $3.99 \pm 1.78$ & 8 & 98190 & AX Cyg & $\mathrm{L}$ & .86 & 5 & 0.70 & 3 \\
\hline 75727 & GO Lup & SRB & 4.3 & V & $4.46 \pm 1.31$ & 0 & 99082 & V1943 Sgr & LB & .82 & 5 & 0.97 & 0 \\
\hline 76377 & R Nor & M & $5.14 \pm$ & V & $2.96 \pm 1.71$ & 20 & 99653 & RS Cyg & SRA & .84 & V & .78 & 1 \\
\hline 76460 & SY CrB & LB & 28 & 5 & $4.21 \pm 1.29$ & 1 & 99990 & RT Cap & SRB & $1.78 \pm 1.48$ & 5 & 0.97 & 7 \\
\hline 77023 & FQ Lup & L: & 0 & 5 & \pm 1.11 & 3 & 0048 & CN Cyg & M & 1.34 & 5 & -0 . & 61 \\
\hline 77027 & BG Ser & M & $-3.78 \pm 2.74$ & 5 & $2.67 \pm 2.05$ & 32 & 100404 & BC Cyg & $\mathrm{L}$ & 2.84 & $\mathrm{~V}$ & .76 & 0 \\
\hline 77284 & $\mathrm{Y} \mathrm{CrB}$ & $\mathrm{L}$ & $4.00 \pm 1.47$ & 5 & $4.77 \pm 1.38$ & 0 & 100582 & V744 Cyg & LB & 26 & 5 & 98 & 32 \\
\hline 78307 & AH Ser & M & $-16.33 \pm 7.71$ & 5 & $-6.84 \pm 5.62$ & 34 & 100605 & UU Dra & SRB & $2.26 \pm 0.87$ & 5 & $3.47 \pm 0.77$ & 0 \\
\hline 78976 & U Ser & M & $2.46 \pm 3.29$ & 5 & $1.82 \pm 2.80$ & 59 & 101888 & RU Vul & SRA & .30 & 5 & 12 & 29 \\
\hline 79233 & RU Her & M & 1.8 & V & $0.81 \pm 1.62$ & 65 & 102082 & V Cyg & $\mathrm{M}$ & 3.65 & 5 & 26 & 11 \\
\hline 80259 & $\mathrm{RY}$ CrB & SRB & 0.9 & V & 1.4 & 31 & 102246 & S Del & $\mathrm{M}$ & -0 & 5 & -0 & 93 \\
\hline 80802 & R UMi & SRB & \pm 0.93 & 5 & $3.85 \pm 0.75$ & 0 & 102829 & T Aqr & M & 1.76 & 5 & -0 & 95 \\
\hline 81309 & X Ara & M & $-2.04 \pm 6.69$ & 5 & $-2.30 \pm 5.46$ & 76 & 104451 & T Cep & M & .75 & 5 & .59 & 0 \\
\hline 81506 & AS Her & M & 5.6 & 5 & 4.93 & 5 & & $\mathrm{~S}$ & M & & 5 & 51 & 0 \\
\hline 81747 & AX Sco & SRB & $4.52 \pm 1.57$ & 5 & $3.67 \pm 1.11$ & 1 & 107516 & EP Aqr & $\mathrm{L}$ & $7.39 \pm$ & 5 & $5.98 \pm$ & 0 \\
\hline 81835 & S Dra & SRB & 2.3 & 5 & $2.46 \pm 0.69$ & 1 & 108183 & V413 Cyg & LB & .08 & 5 & .84 & 30 \\
\hline 82912 & RR Sco & M & 2.8 & 5 & $3.10 \pm 1.16$ & 4 & 108588 & OU Peg & $\mathrm{Ll}$ & .92 & 5 & 2.40 & 4 \\
\hline 84213 & TT Dra & SRB & $1.05 \pm 0.90$ & 5 & $0.82 \pm 0.84$ & 43 & 109070 & SV Peg & SRB & $5.10=$ & 5 & $=1.03$ & 9 \\
\hline 84346 & V438 Oph & SRB & $-1.25 \pm 1.41$ & 5 & $-0.04 \pm 1.20$ & 98 & 109089 & RZ Peg & M & $3.54 \pm 1.36$ & $\mathrm{~V}$ & $3.23 \pm 1.23$ & 9 \\
\hline 84833 & V656 Her & LB: & $6.90 \pm 0.79$ & 5 & $6.55 \pm 0.71$ & 0 & 110146 & $X$ Aqr & M & $-4.01 \pm 5.73$ & 5 & $1.88 \pm 3.77$ & 76 \\
\hline 85617 & TW Oph & SRB & $3.57 \pm 1.34$ & 5 & $1.12 \pm 0.97$ & 52 & 110478 & $\pi^{1}$ Gru & $\mathrm{L}$ & $6.54 \pm 1.01$ & 5 & $5.01 \pm 0.95$ & 0 \\
\hline 86836 & Z Oct & M & $-1.18 \pm 3.04$ & 5 & $1.02 \pm 1.95$ & 68 & 110948 & V410 Lac & LB & $1.28 \pm 1.06$ & 5 & $2.16 \pm 0.89$ & 6 \\
\hline 87063 & SX Sco & $\mathrm{L}$ & $2.75 \pm 1.42$ & 5 & $2.47 \pm 1.13$ & 11 & 111043 & $\delta^{2} \mathrm{Gru}$ & LB: & $10.04 \pm 1.11$ & $\mathrm{~V}$ & $10.08 \pm 0.96$ & 0 \\
\hline 87190 & V337 Her & SRB & $3.12 \pm 0.76$ & 5 & $2.34 \pm 0.75$ & 2 & 112784 & SX Peg & M & $2.12 \pm 2.99$ & 5 & $4.81 \pm 2.28$ & 11 \\
\hline 88397 & OU Her & LB & $-0.58 \pm 1.92$ & 5 & $0.12 \pm 1.62$ & 95 & 112868 & AF Peg & SRB & 0.93 & 5 & $1.93 \pm$ & 26 \\
\hline 88838 & VX Sgr & SRC & $3.03 \pm 1.95$ & 5 & $1.86 \pm 1.75$ & 53 & 112961 & $\lambda \mathrm{Aqr}$ & $\mathrm{L}$ & $8.33 \pm 1.13$ & $\mathrm{~V}$ & $8.82 \pm 0.91$ & 0 \\
\hline 89568 & RY Oph & M & $3.17 \pm 1.95$ & 5 & $3.46 \pm 1.54$ & 16 & 113131 & HR Peg & SRB & $3.37 \pm 0.94$ & 5 & $2.05 \pm 0.79$ & 5 \\
\hline 89739 & RS Tel & $\mathrm{RCB}$ & $3.00 \pm 2.98$ & 5 & $4.74 \pm 2.78$ & 21 & 114017 & ER Aqr & LB & $4.10 \pm 1.26$ & 5 & $4.40 \pm 1.12$ & 0 \\
\hline 89980 & V4028 Sgr & SR: & $2.80 \pm 1.11$ & 5 & $2.76 \pm 1.03$ & & 114757 & TY And & SRB & $0.87 \pm 1.32$ & 5 & $1.37 \pm 1.20$ & 43 \\
\hline 90709 & SS Sgr & SRB & $5.92 \pm 2.86$ & V & $6.21 \pm 2.77$ & & 115188 & W Peg & M & $3.46 \pm 1.38$ & 5 & $3.36 \pm 1.14$ & 4 \\
\hline 90883 & T Lyr & $\mathrm{L}$ & $1.58 \pm 0.75$ & 5 & $2.13 \pm 0.64$ & & 116018 & & $\mathrm{LC}$ & $1.64 \pm 1.05$ & 5 & $1.25 \pm 0.92$ & 30 \\
\hline 93605 & SU Sgr & SR & $3.14 \pm 1.44$ & V & $1.45 \pm 1.36$ & 25 & 116681 & ST And & SRA & $5.50 \pm 2.83$ & 5 & $-0.07 \pm 1.79$ & 97 \\
\hline 93820 & R Aql & M & $4.73 \pm 1.19$ & 5 & $3.96 \pm 0.94$ & 1 & 118188 & R Cas & M & $9.37 \pm 1.10$ & 5 & $10.04 \pm 1.10$ & 0 \\
\hline
\end{tabular}

where $N$ is the number of data points and $p$ the number of parameters in the model ( $p=5$ for a single-star solution, and $p=7$ for a VIM solution). Defining

$\alpha=\operatorname{Pr}[\hat{F}<F(1, N-p) \mid \varpi=0]$,

a non-zero parallax is considered significant if, say, $\alpha \leq 5 \%$ which roughly corresponds to parallaxes with $\varpi / \sigma_{\varpi}>2$. The value of $\alpha$ is listed in Table 1 . Eighty objects satisfy the criterion $\alpha \leq 5 \%$.

\section{Discussion}

There are several possible ways to check the validity of the new results:

1. The revised parallax may be compared to that estimated by Mennessier \& Luri (2001) using a maximum likelihood approach incorporating kinematic and photometric data, distribution functions and a model of the Galaxy.

2. The revised parallax may be compared to estimates based on the period-luminosity relationship for Mira variables. 


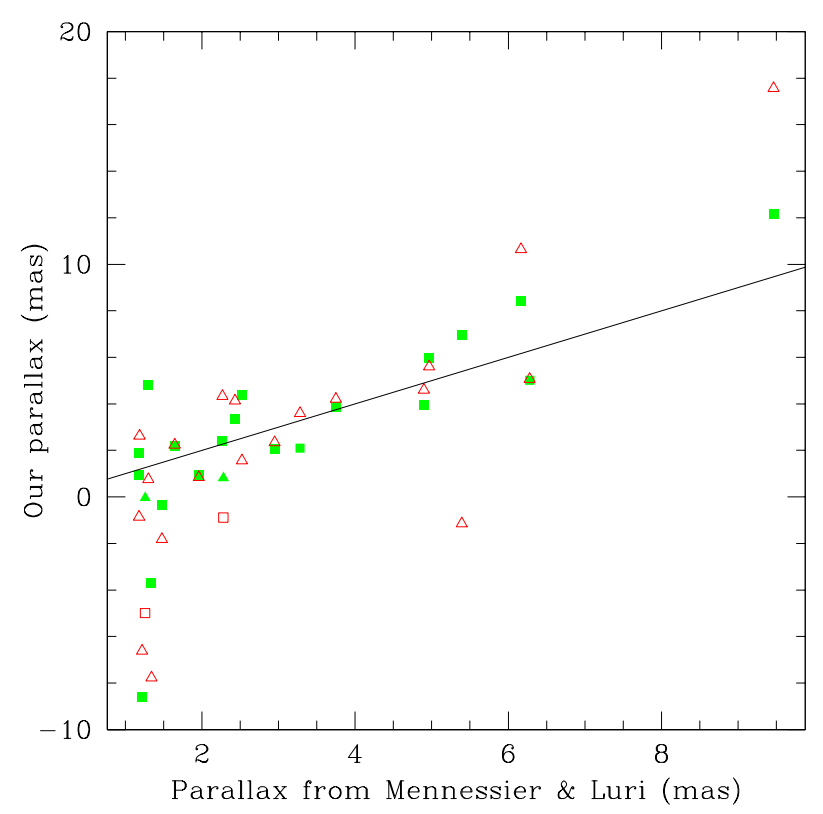

Fig. 3. Comparison of the parallaxes from Mennessier \& Luri (2001) with those obtained with a VIM model (triangles) or with a singlestar model (squares). Filled symbols represent the adopted model at the $0.27 \%$ confidence level (i.e., for a VIM solution, $F_{D}>3.44$ in Eq. (2)). The diagonal is represented by a solid line.

3. A direct confirmation that VIM solutions indeed correspond to visual binaries should be seeked. Conversely, it should be checked whether the known visual binaries among LPVs indeed behave as VIM when they are not resolved bt Hipparcos.

Each of these items is discussed in turn in the remainder of this section.

\subsection{Comparison with Mennessier \& Luri parallaxes}

Mennessier et al. (2001) used a maximum likelihood method to estimate distances of long-period variable stars, based on kinematic and photometric data, and assuming a priori functional relationships describing the Galaxy and the stellar sample (exponential distribution of the number of stars perpendicular to the galactic plane, gaussian distribution of the absolute magnitudes, Schwarzschild velocity ellipsoid). Among the stars studied by Mennessier \& Luri (2001), 23 also appear in our sample of confirmed Hipparcos VIM stars for which the epoch $V-I$ indices are now available. The absolute $K$ magnitudes listed by Mennessier \& Luri (2001) have been converted to parallaxes using apparent $K$ magnitudes from the literature (see Gezari et al. 1999; Whitelock et al. 2000), neglecting interstellar extinction and circumstellar reddening. These "astrophysical" parallaxes are compared in Fig. 3 with the reprocessed single-star or VIM solutions. Except for parallaxes lower than 2 mas, where the Hipparcos data are of too poor a quality to derive meaningful parallaxes, the single-star solution is generally closer to the estimated "astrophysical" parallax than the VIM solution. Rejecting the VIM solution in those cases requires a much more severe confidence level than the $10 \%$ level adopted so far. It is found that a level at $0.27 \%$
Table 2. The 19 stars flagged as VIM in the Hipparcos catalogue, and with $(V-I)_{\mathrm{H} 75}>5.0$. The $(V-I)_{\mathrm{H} 75}$ index is compared to the best value of $V-I$ available at the time of the publication of the Hipparcos and Tycho Catalogues (field $\mathrm{H} 40$ ) and to the range of $V-I$ used in the chromaticity corrections. Among these 19 stars, only HIP 2215, HIP 68815 and HIP 93605 remain VIM at the $0.27 \%$ confidence level after our improved reprocessing

\begin{tabular}{lccccccc}
\hline \hline HIP & H75 & H40 & $V-I$ & HIP & H75 & H40 & $V-I$ \\
\hline 2215 & 5.77 & 3.91 & $3.68-3.80$ & 87190 & 5.34 & 3.74 & $3.35-3.74$ \\
12193 & 5.92 & 1.15 & $2.26-4.81$ & 93605 & 6.66 & 4.32 & $4.25-4.42$ \\
44995 & 5.15 & 5.15 & $3.95-4.71$ & 97629 & 6.13 & 6.13 & $4.04-6.28$ \\
47886 & 5.15 & 3.41 & $4.49-5.80$ & 98031 & 5.34 & 4.94 & $4.33-5.05$ \\
48036 & 9.03 & 9.03 & $3.97-5.92$ & 99082 & 5.39 & 4.87 & $4.51-4.79$ \\
67419 & 9.29 & 5.36 & $4.86-5.56$ & 100605 & 5.34 & 4.49 & $4.25-4.48$ \\
68815 & 5.88 & 4.10 & $3.64-4.02$ & 108588 & 5.51 & 5.51 & $3.64-3.79$ \\
70969 & 7.60 & 4.60 & $4.36-4.65$ & 110478 & 6.51 & 4.65 & $3.99-4.30$ \\
71802 & 5.79 & 4.09 & $3.76-4.03$ & 118188 & 5.34 & 5.34 & $4.02-6.03$ \\
77284 & 5.34 & 5.34 & $3.77-4.11$ & & & & \\
\hline
\end{tabular}

(corresponding to $F_{D}>3.44$ ) prevents from adopting the VIM solutions lying far off the diagonal in Fig. 3. Incidentally, we note that this level is the same as that used in the Hipparcos catalogue to accept a DMSA/G solution (i.e., a model with an acceleration term). At the $0.27 \%$ confidence level, only three VIM solutions pass the test: HIP 36669, 79233, and 94706. To our knowledge, none of these is known to be a visual binary, but all three have small ( $<1$ mas), non-meaningful parallaxes.

With this much higher confidence level, only 27 VIM solutions are retained (as listed in Table 1), i.e., just $14 \%$ of the original sample (Figs. 1c, d). We will show in Sect. 5.3 that this more stringent confidence level appears fully justified also for pre-main sequence binaries.

To conclude, it should be noted that the Mennessier \& Luri (2001) analysis provides a way to evaluate the accuracy of our revised parallax for HIP 99082, which differs by more than $3 \sigma$ from the Hipparcos value. Based on their maximum-likelihood estimate for $M_{K}(-8.03)$ and the $2 \mu \mathrm{m}$ sky-survey $K$ magnitude $(-1.41$; Neugebauer \& Leighton 1969), a parallax $\varpi=4.7$ mas is derived, in good agreement with our reprocessed value of $\varpi=5.02 \pm 0.97$ mas, which both differ significantly from the Hipparcos value $\varpi_{\text {HIP }}=1.81 \pm 0.84$ mas.

\subsection{Period-luminosity relation}

The period-luminosity (P-L) relation proposed by Whitelock $\&$ Feast (2000) for long-period variables may be used to evaluate the quality of our revised parallaxes, although this argument to some extent is circular, since the Whitelock \& Feast's $\mathrm{P}-\mathrm{L}$ relation is based on Hipparcos data.

Nevertheless, the P-L relation provides a very useful diagnostic for HIP 65835 (= R Hya). The Hipparcos VIM solution yields $\varpi=1.62 \pm 2.43$ mas, to be compared with $8.44 \pm 1.00$ for our reprocessed single-star model, which lies many $\sigma$ away from the Hipparcos value! Which value then is the correct one? 
Based on their P-L relation and new $K$ magnitudes, Whitelock et al. (2000) derive a distance of $0.14 \mathrm{kpc}(\varpi=7.14$ mas) for $\mathrm{R}$ Hya which agrees (to within $1.3 \sigma$ ) with our new parallax.

Similar cases are HIP 1901 (=R And) and HIP 77027 (= BG Ser), with $\varpi_{\text {HIP,VIM }}=-0.06 \pm 6.49$ mas and $-3.78 \pm$ 2.74 mas, as compared to $6.96 \pm 3.63$ mas and $2.67 \pm 2.05$ mas, respectively, for our revised single-star solutions. For R And, the P-L relation yields $\varpi=2.3$ mas (Whitelock et al. 2000), $1.3 \sigma$ away from our revised estimate, and for BG Ser, 1.92 mas, or $0.4 \sigma$ away. HIP 1834 (= T Cas) is another case where the revised parallax $(\varpi=3.08 \pm 0.90)$ is in agreement with both the P-L distance estimate $(\varpi=3.45$ mas) and the Mennessier \& Luri (2001) value ( $\varpi=2.48$ mas, from $M_{K}$ and the $2 \mu \mathrm{m}$ skysurvey $K$ magnitude, Neugebauer \& Leighton 1969), despite being very different from $\varpi_{\text {HIP }}=0.59 \pm 1.07$ mas.

Thus when large discrepancies exist between the reprocessed and the Hipparcos parallaxes, the reprocessed parallax appears quite satisfactory. A more thorough re-investigation of the P-L relation will be presented in a separate paper (Knapp et al. 2003).

\subsection{Known visual binaries}

The VIM model does not provide the angular separation between the primary and secondary, unless the magnitude of the latter is known. Therefore only the position angle $(\theta)$ can be derived in all cases as

$\tan \theta=\frac{D_{\alpha^{*}}}{D_{\delta}}$

where $D_{\alpha^{*}}$ and $D_{\delta}$ have been defined in relation with Eq. (2). Our rederived value for the position angle and the Hipparcos one agree fairly well (Fig. 4), except for HIP 67410.

\subsubsection{Pre-main sequence stars}

Bertout et al. (1999) critically evaluated the eight pre-main sequence stars flagged as VIM in the Hipparcos catalogue, with $F_{D}$ ranging from 2.23 to 9.08 . Only four of these were known to be binaries before Hipparcos. Among them, only HIP $20777=$ DF Tau $\left(F_{D}=2.79\right)$ would be discarded with the threshold on $F_{D}$ set to 3.44. Bertout et al. (1999) indeed confirm that the VIM solution is not entirely satisfactory. On the other hand, at that confidence level, only HIP $100289=$ V1685 Cyg $\left(F_{D}=3.96\right)$ would remain a newly discovered binary. In conclusion, the more stringent criterion on $F_{D}$ suggested from the study of long-period variables (Sect. 5.1) is perfectly acceptable for pre-main sequence stars as well, since most of the genuine binaries are accepted VIM solutions.

\subsubsection{Long-period variables}

Among the 188 objects listed in Table 1, not many are known to be genuine close visual binaries (see Jorissen 2003 for a complete list of binaries involving long-period variables).

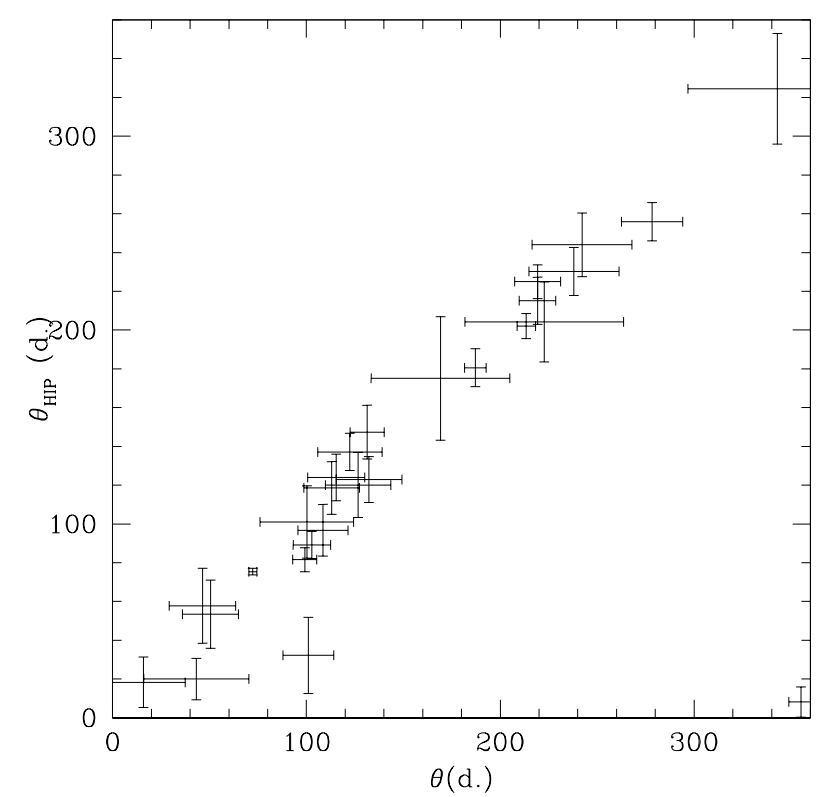

Fig. 4. Position angles with the old and new VIM solutions. HIP 67410 is the only case where the two angles are different but the parallax is small anyway.

There first is a group a five close visual binaries which are indeed accepted as VIM:

HIP 91389 (= X Oph = WDS 18384+0850). Although X Oph does not appear in Table 1 (because the Hipparcos catalogue flags it as a stochastic solution DMSA/X), it is almost a textbook case. It has a variation range $H p=5.8$ to 8.1 (5th to 95 th percentile), and a $V=8.6$ companion located $0 . ' 4$ away at a position angle of $131^{\circ}$. The confidence level of our VIM solution is very high $\left(F_{D}=72.4\right)$, and is in very good agreement with the ground based data, since it yields a position angle of $147^{\circ}$ and a separation of 0.328 . The only worry about this solution is its negative parallax, and the large residuals (20 mas), indicative that there is some other effect present, possibly the orbital motion (although the estimated orbital period $-485 \mathrm{y}$ - is quite long with respect to the duration of the Hipparcos mission; Hartkopf et al. 2001).

HIP 46806 (= R Car = WDS 09322-6247). A close visual binary with a $V=11.3$ companion located at $\rho=1{ }^{\prime \prime} 8$ and $\theta=140^{\circ}$, with an accepted VIM reprocessed solution $\left(F_{D}=6.48\right)$. Despite the high confidence level of the VIM solution, it yields a position angle $\theta=355^{\circ} \pm 7^{\circ}$ in disagreement with the ground-based solution.

HIP 79233 (=RU Her). This star is suspected to be a binary by Herbig (1965) who notes that the absorption features are remarkably weak, as though veiled, the spectrum may be composite. With $F_{D}=3.53$, the VIM solution is indeed adopted.

HIP 93605 (= SU Sgr = WDS 19037-2243). This star is listed by Proust et al. (1981) as having a $V=13.3$ companion at $\rho=11^{\prime \prime} 1$ and $\theta=239^{\circ}$. The semiregular variable SU Sgr varied between $H p=7.3$ and 7.9 (5th and 95th percentiles, fields $\mathrm{H} 49$ and $\mathrm{H} 50$ ) during the Hipparcos mission. Using the formulae of Wielen (1996), we find that, 
in such conditions, the photocenter should exhibit a back and forth motion with a total amplitude of 3.3 mas. This motion is probably marginally detectable by Hipparcos, except that the companion may be below the photometric detection threshold. The position angle of the companion with respect to the variable star obtained from the VIM solution $\left(\theta=102^{\circ} \pm 7^{\circ}\right)$ is, however, not consistent with the groundbased position angle $\left(\theta=239^{\circ}\right)$, thus casting a doubt on the reality of the VIM solution.

HIP 94706 (=T Sgr). This star is also noted by Herbig (1965) as having a composite spectrum, later confirmed by Culver \& Ianna (1975). Although it is flagged as VIM in the Hipparcos catalogue, both the Hipparcos and our reprocessed parallaxes (also obtained within the framework of a VIM model) are negative by a large amount. There must thus be another effect disturbing the VIM solution, perhaps an orbital motion (the orbital period is still unknown).

The discrepancy between ground-based and Hipparcos results for HIP 46806 and 93605 may result from the 1'.2 periodicity of the focal-plane grid, thus limiting the effectiveness of the VIM model to systems with separations below 0.'5.

There is then a group of 3 stars which are close visual binaries, but not accepted as VIM. The two carbon semiregular variable stars with composite spectra HIP 27135 (=TU Tau) and HIP 86873 (=SZ Sgr; Olson \& Richer 1975) were neither flagged as VIM by the Hipparcos catalogue, nor by our reprocessing. The VIM nature of HIP $110478\left(=\pi^{1} \mathrm{Gru}\right)$ is not confirmed by our reprocessing, but the companion is indeed rather far away (2'.7). We may thus conclude that the number of genuine binaries not detected as VIM is indeed small.

Finally, several stars not accepted as VIM in Table 1 are seen as single, as it should be, by speckle observations having a resolution of about 50 mas: HIP 63950 (Mason et al. 1999), HIP 1834, 35045, 95173, 109070, 110948, 112868, and 115188 (Mason et al. 2001).

HIP 36288 (= Y Lyn) is the only puzzling case, since despite being confirmed as VIM by our reprocessing, it could not be resolved by Mason et al. (2001). The only noteworthy property of this star possibly relevant in the present context is its spatial extension in the IRAS 60 and $100 \mu \mathrm{m}$ passbands (Young et al. 1993).

\section{Conclusion}

Instantaneous $V-I$ indices derived from a color transformation based on $\mathrm{Hp}-V_{\mathrm{T} 2}$ have been used to improve the chromaticity correction of red and variable stars. All stars flagged as Variability-Induced Mover (VIM) in the Hipparcos catalogue have been reprocessed. It turns out that about $85 \%$ of those objects can now be satisfactory modeled with the basic 5-parameter, single-star solution. This result explains why many of the Hipparcos VIM solutions did not receive confirmation of their binary nature from speckle observations.

Acknowledgements. We thank L. Lindegren for valuable suggestions and for providing us the unpublished NDAC chromaticity constants, C. Dettbarn for technical details about the actual processing of the VIM by the FAST consortium, and C. Fabricius for his help with the Tycho-2 photometry. This research was supported in part by ESA/PRODEX C15152/01/NL/SFe(IC) and by NASA via grant NAG5-11094.

\section{References}

Bertout, C., Robichon, N., \& Arenou, F. 1999, A\&A, 352, 574 Culver, R. B., \& Ianna, P. A. 1975, ApJ, 195, L37

ESA. 1997, The Hipparcos and Tycho Catalogues (ESA SP-1200)

Gezari, D. Y., Pitts, P. S., \& Schmitz, M. 1999, Catalog of Infrared Observations, 5th ed.

Hartkopf, W. I., Mason, B. D., \& Worley, C. E. 2001, AJ, 122, 3472

Herbig, G. H. 1965, Kleine Veröff. Remeis-Sternwarte, 164

Høg, E., Fabricius, C., Makarov, V. V., et al. 2000, A\&A, 355, L27

Jorissen, A. 2003, in Asymptotic giant branch stars, ed. H. Habing, \& H. Olofsson (New York: Springer Verlag)

Kholopov, P. N., Samus, N. N., Frolov, M. S., et al. 1998, Combined General Catalogue of Variable Stars, 4th ed.

Knapp, G. R., Pourbaix, D., Platais, I., \& Jorissen, A. 2003, A\&A, in preparation

Lindegren, L., Mignard, F., Söderhjelm, S., et al. 1997, A\&A, 323, L53

Mason, B. D., Hartkopf, W. I., Holdenried, E. R., \& Rafferty, T. J. 2001, AJ, 121, 3224

Mason, B. D., Martin, C., Hartkopf, W. I., et al. 1999, AJ, 117, 1890

Mennessier, M. O., \& Luri, X. 2001, A\&A, 380, 198

Mennessier, M. O., Mowlavi, N., Alvarez, R., \& Luri, X. 2001, A\&A, 374, 968

Neugebauer, G., \& Leighton, R. B. 1969, Two-Micro Sky Survey Catalogue (NASA SP-3047)

Olson, B. I., \& Richer, H. B. 1975, ApJ, 200, 88

Platais, I., Pourbaix, D., Jorissen, A., et al. 2003, A\&A, 397, 997

Proust, D., Ochsenbein, F., \& Pettersen, B. R. 1981, A\&AS, 44, 179

Whitelock, P., \& Feast, M. 2000, MNRAS, 319, 759

Whitelock, P., Marang, F., \& Feast, M. 2000, MNRAS, 319, 728

Wielen, R. 1996, A\&A, 314, 679

Young, K., Phillips, T. G., \& Knapp, G. R. 1993, ApJS, 86, 517 